\title{
Surface Reconstruction and Decahedral Structure of Bimetallic Nanoparticles
}

\author{
J. L. Rodríguez-López, ${ }^{1}$ J. M. Montejano-Carrizales, ${ }^{2}$ U. Pal, ${ }^{3,4}$ J. F. Sánchez-Ramírez, ${ }^{5}$ H. E. Troiani, ${ }^{6}$ D. García, ${ }^{7}$ \\ M. Miki-Yoshida, ${ }^{7,8}$ and M. José-Yacamán ${ }^{7, *}$ \\ ${ }^{1}$ Advanced Materials Department, IPICYT, Camino Presa San José 2055, 78216 San Luis Potosí, San Luis Potosí, Mexico \\ ${ }^{2}$ Instituto de Física, Universidad Autónoma de San Luis Potosí, 78000 San Luis Potosí, San Luis Potosí, Mexico \\ ${ }^{3}$ Instituto de Física, Universidad Autónoma de Puebla, Apartado Postal J-48, 72570 Puebla, Puebla, Mexico \\ ${ }^{4}$ Instituto Mexicano del Petróleo, Eje Central Lázaro Cardenas 152, 07730 México, Distrito Federal, Mexico \\ ${ }^{5}$ Facultad de Ciencias Químicas, Instituto de Ciencias, Universidad Autónoma de Puebla, 72570 Puebla, Puebla, Mexico \\ ${ }^{6}$ Instituto Balseiro and CNEA 8400, San Carlos de Bariloche, Rio Negro, Argentina \\ ${ }^{7}$ Department of Chemical Engineering and Texas Materials Institute, The University of Texas, 78712-1063 Austin, Texas, USA \\ ${ }^{8}$ Centro de Investigación en Materiales Avanzados S.C., Miguel de Cervantes 120, 31109 Chihuahua, Chihuahua, Mexico
}

(Received 20 September 2003; published 13 May 2004)

\begin{abstract}
We report on energetic surface reconstruction phenomena observed on bimetallic nanoparticle systems of $\mathrm{AuPd}$ and $\mathrm{AuCu}$, similar to a resolidification effect observed during the cooling process in lead clusters. These binary alloy nanoparticles show the fivefold edges truncated, resulting in $\{100\}$ facets on decahedral structures, an effect largely envisioned and reported theoretically, with no experimental evidence so far. We demonstrate experimentally as well as by computational simulations that this new eutectic structure is favored in such nanoalloy systems.
\end{abstract}

DOI: 10.1103/PhysRevLett.92.196102

PACS numbers: 68.35.Bs, 81.07.-b

There is growing evidence that clusters and nanoparticles have a shell periodicity and that they grow by the successive accumulation of layers [1]. Their growth is then related to one of the oldest mathematical problems, the packing of spheres in the densest arrangement, i.e., the perfect tetrahedral packing, which is a nonfilling space structure. Noncrystalline structures with fivefold axes of symmetry, such as icosahedrons and decahedrons, are composed of deformed tetrahedral units where adjacent tetrahedral faces meet at a twinning plane. Thus, the growth of noncrystalline nanoparticles and clusters can proceed only with the introduction of defects such as disclinations [2], which lead to polytetrahedral structures [3] or with close packing schemes, which give well defined structures such as the Mackay icosahedron [4], the truncated decahedron [5], and tetrahedron [6]. Recently, other structures such as the truncated icosahedron [7] and the double icosahedron [8] have been found experimentally, and particularly interesting are the clusters and nanoparticles with pentagonal symmetry, which have been the subject of very intense research both experimentally $[9,10]$ and theoretically [11-13].

When metal nanoparticles are synthesized, irrespective of the method of preparation, they can have different shapes like square, quasispherical, and pentagonal. Those shapes can be associated in three dimensions to the pyramid, the icosahedral, and the decahedral structure, respectively. We have found that bimetallic structures with fivefold symmetry have a number of structural variants and some unexpected pentagonal shapes that cannot be obtained by simply rotating the main symmetry axis at all, but by displacements of the fivefold axis from the center of the particle, which generates asymmetric structures [14]. Those variations can be understood in terms of the concept of the energy landscape scenario, which implies that for a given particle size many other structures (or isomers) have a similar total energy and therefore a jump between different states is very likely in this shallow potential energy surface. This has been reported by Iijima [15] and Ajayan [16] for quasimelting states where a metallic nanoparticle changes its shape in a very dramatic way under the electron beam irradiation. This effect is a direct proof of the energy landscape concept.

We have studied the structure of bimetallic nanoparticles of AuPd and AuCu both experimentally and theoretically and present experimental evidence that two of the well known defects in solid state materials that influence surface stability, i.e., vacancies and surface reconstruction that tend to reduce the free energy of the systems, are present in our particles, and thus these defects stabilize the structures of our bimetallic nanosystems. The formation of vacancies in the interior and on the surface of the cluster stabilize icosahedral structure, and has been demonstrated theoretically earlier for pure noble metal clusters [17]. These vacancies imply important modifications on the diffusion profiles in metallic clusters (due to the migration of vacancies), and the consequence is noticeable in the chemical ordering in bimetallic clusters [18]. Surface reconstruction is induced by the binary nature of the system, mainly due to this chemical ordering. Our extensive experiments on $\mathrm{AuPd}$ and $\mathrm{AuCu}$ nanoclusters reveals that at a given composition (Au50/Pd50 in case of AuPd clusters), we obtain a high rate of formation of these unusual structures with fivefold although nonperfect symmetry and report on surface reconstruction phenomena in these systems. We performed molecular dynamics (MD) simulations on bimetallic systems and propose a new structure with 
fivefold symmetry and a reconstructed surface layer that corresponds to the experimentally observed nonperfect pentagonal structures.

Colloidal dispersion of the AuPd bimetallic clusters was prepared by simultaneous reduction of the two metal ions in a water/methanol $(v / v=1 / 1)$ solvent in the presence of poly( $N$-vinyl-2-pyrrolidone). Ethanol in the presence of water acted as a reducing agent. In the case of monometallic Au colloids, the methanol solution of gold ions was reduced by $\mathrm{NaOH}$. Details of the sample preparation process is given elsewhere [19]. Images of high resolution transmission electron microscopy (HRTEM) were obtained using a JEOL-2010-FEG microscope, with the resolution of $0.19 \mathrm{~nm}$ and a tilting possibility of $\pm 30^{\circ}$, and a JEOL 4000EX with a resolution of $0.17 \mathrm{~nm}$. HRTEM images of nanoparticles were obtained at the first optimum defocus (Scherzer focus) or at the second optimum defocus condition, resulting in images of atomic columns of black (first maximum) or white (second maximum) dots. In order to compare with our experimental results, molecular dynamics simulations on bimetallic clusters were done using a Sutton-Chen (SC) [20] many-body interatomic potential in the constant temperature ensemble $(N V T)$, with a Verlet algorithm for the integration of Newton's equations of motion and a time step of $5 \mathrm{fs}$. A cutoff ratio of $7.0 \AA$ for the range of atomic interaction was set in all the cases and the NoseHoover thermostat is applied with a relaxation time of $10 \mathrm{fs}$. The use of the simple SC [20] potential leads to an accurate description of many properties of fcc metals and their alloys [21], and in the present work we have applied this potential to model the interaction in the $\mathrm{AuCu}$ alloy clusters. For details of the simulation process, the readers can consult Ref. [18]. Simulated HRTEM images at Scherzer focus were obtained for the models presented here, their comparison with experimental results allows us to interpret and elucidate the experimental images.

For experimental and simulation analysis we considered clusters of monometallic $\mathrm{Au}, \mathrm{Pd}$, and bimetallic nanoparticles of $\mathrm{Au}_{5} \mathrm{Pd}, \mathrm{AuPd}, \mathrm{AuPd}_{5}$ nominal compositions. It was found that the decahedral structure was the most common type of particle observed, although with significant deviations and defects with respect to the perfect decahedral structure. Particles shown in Figs. 1(a) and 1(b) correspond to the typical cases. First of all, we note the missing atom in the center of these bimetallic particles. This feature was observed frequently in our experiments. For larger particles, several surface vacancy sites are observed, as can be seen in Fig. 1(b). A processed image (noise reduction filter applied) and its corresponding diffraction pattern for a typical particle are shown in Figs. 1(c) and 1(d), respectively, where the bimetallic contrast and decahedral structure are evident.

A second experimental example for a AuPd particle supported on amorphous carbon are shown in Figs. 2(a) and 2(b). The simulated HRTEM image [Fig. 2(c)] corresponds with the model for a particle with surface re-
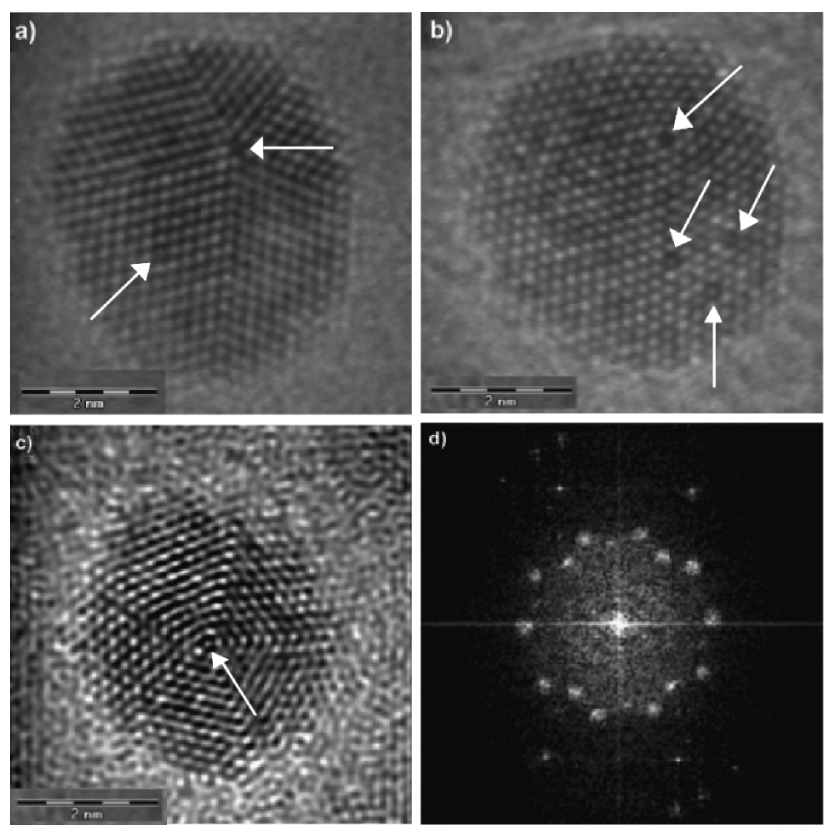

FIG. 1. Different asymmetric fivefold structures for bimetallic nanoparticles. (a),(b) The surface vacancies (pointed to by arrows) characteristic of these bimetallic systems. (c) A particle with a central vacancy site, and (d) the corresponding diffraction pattern where the decahedral structure is evidenced. The bar line measures $2 \mathrm{~nm}$ in all the images.

construction, Fig. 2(d). This type of structural arrangement has been obtained by means of MD by Hendy et al. [22] on lead clusters. A model for a decahedral particle with surface reconstruction is presented in Fig. 2(e). The structure results with the outer layer of atoms in hexagonal close packed (hcp) surface sites, a surface reconstruction that improves the energetic stability of these binary particles. In these particles, the normal fcc atomic growth sequence ...ABC becomes ...ABCB, which is a surface reconstruction pointed out by Hendy et al. [22] on lead clusters after a resolidification process. The first point to be noted is that the above-mentioned fact results in a missing atom in the central region where all the tetrahedrons meet [Fig. 2(e)]. This atom is loosely bonded because of the lower coordination at this site. And, second, this hcp packing in the last atomic layer results on $\{100\}$ facets distributed in the surface. Vacancies formation favoring icosahedral structures on metallic clusters had been studied by Mottet et al. [17] and surface reconstruction (truncated edges on the icosahedron) that improves the energy stability of multitwinned particles on a wide range of sizes by Hendy et al. [22]. Both studies used MD simulations.

Final experimental examples are given in Fig. 3. Again we have another particle (left) that has faces $\{100\}$ and $\{111\}$ as well as a missing atom at the center. The good contrast in all the high resolution images indicate the good contact between the particles and the substrate to avoid electrostatic charging effect. The image at the right shows a decahedral faceted particle with another defect. 


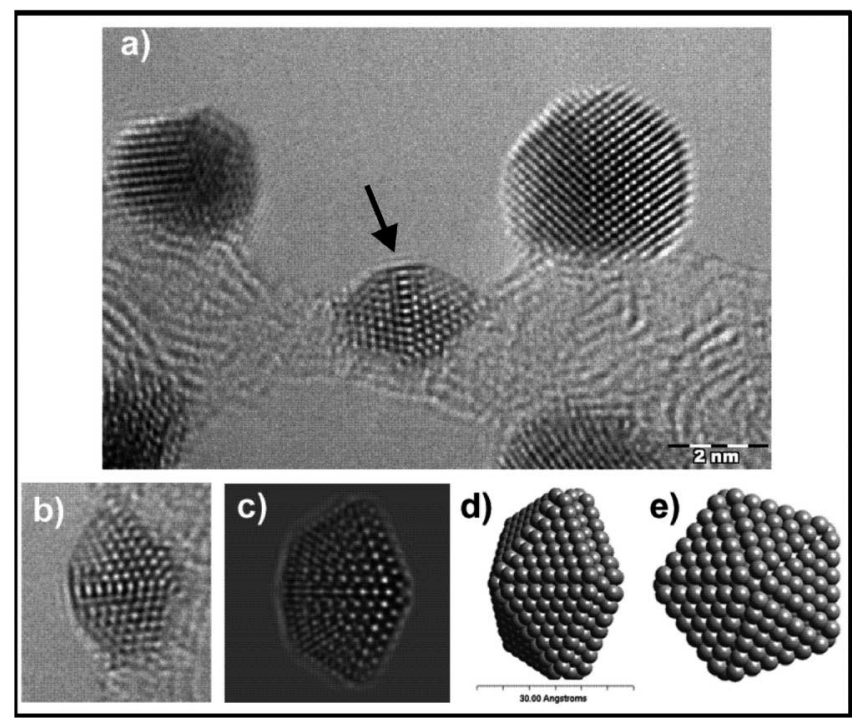

FIG. 2. (a) Pointed to by the arrow and (b) shows high resolution images for a decahedral AuPd particle presented in our experiments. This kind of particle is compared with the simulated HRTEM image at the proper orientation in (c) using the model in (d). A model for the particle where surface reconstruction occurs is presented in (e). The model is a regular pentadecahedra where the last layer of atoms does not follow the fcc $(\ldots A B C)$ sequence. The faulted packing (hcp) in the last layer is reconstructed due to energy considerations of stability.

Two faces with (111) orientation are forming a groove, and a (100) plane is formed. The resulting defect is similar to the chevron defect reported by Radetic et al. [23].

We believe that different atomic ratios between atoms involved in binary systems (e.g., AuPt, AuRh, and PtPd) would give similar results because formation and migration of vacancies are favored when the atomic aspect ratio is different from the unit. Also, at least in the small size regime $(2-5 \mathrm{~nm})$, we expect to find surface reconstruction because icosahedral and decahedral structures are the most energetically favored for metallic systems.

The cohesion energy for surface reconstructed decahedrons using the embedded atom method (EAM) in
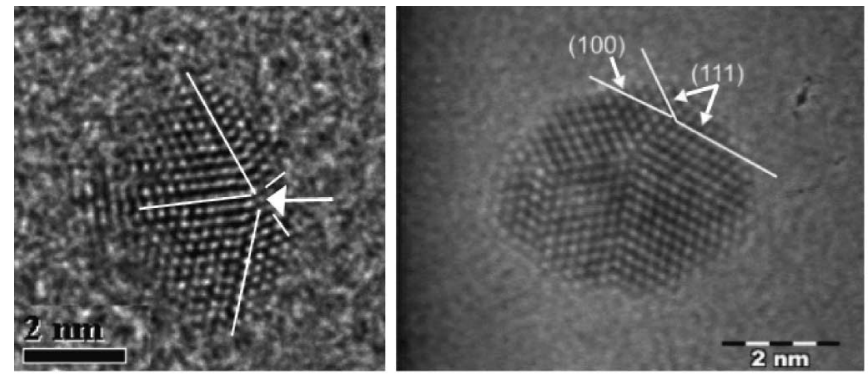

FIG. 3. Bimetallic AuPd nanoparticles that show (left) $\{100\}$ and $\{111\}$ facets and a missing atom at the center site (pointed to by the arrow). The pentagonal symmetry has been marked with lines. At the right, irregular decahedral structure due to the displacement of the main fivefold symmetry axis from the center of the particle and a chevron defect.
Foiles' version [24] was calculated for palladium clusters. The results are presented in Fig. 4. As can be seen, the relative stability of this structural growth pattern is as high as the cubo-octahedral and the icosahedral growth patterns. This high stability is explained by the large surface vacancy sites and the truncated fivefold edges, as has been reported theoretically before by Marks [5] and Hendy et al. [22], and justifies the large number of particles with these features in our bimetallic systems.

Molecular dynamics simulations were done for bimetallic clusters of AuPd and $\mathrm{AuCu}$, and the same physical trends are valid for both systems. These results are summarized in Fig. 5. It is clear that the evolution in the structure of the particle depends on the concentration of the components and on the number of atoms. Figure 5(a) shows a system with 561 atoms at $10 \% \mathrm{Cu}$ concentration, where we observe that the copper atoms group together, although there is not enough concentration for a nucleation process. When the copper atom concentration is greater than $10 \%$, noncrystalline structures like pseudo-icosahedrons [Fig. 5(b), 561 atoms, 50\% copper], icosahedrons [Fig. 5(c), 923 atoms, 25\% copper], and the core-shell [Fig. 5(d), 923 atoms, 50\% copper] structure are obtained. In all the cases, we noted copper atoms migrating to the core, as was evidenced by the diffusion coefficient analysis [18] forming the core-shell structure in agreement with the observation of Srnová-Šloubová et al. [14]. The interesting point is that the presence of two noble metals increases the particle morphology density of the complex potential energy surface. It starts as a fcc cubo-octahedral particle, and it is transformed to a

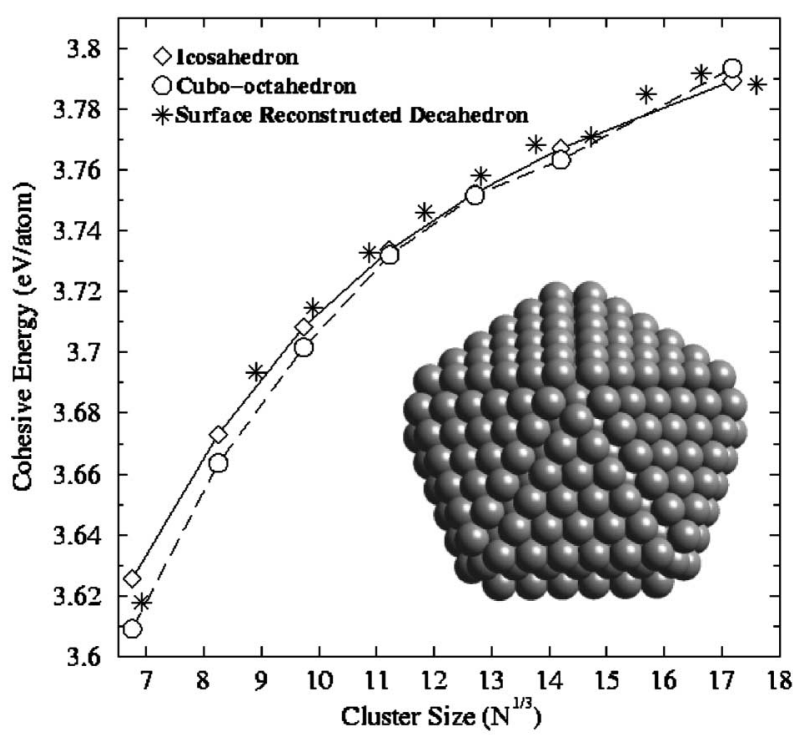

FIG. 4. Cohesion energy versus particle size for the structure with surface reconstruction shown in Fig. 2 and in this inset, calculated by means of the EAM using Pd parameters. For reference, we have plotted the results for the most known stable structure families at this size range, i.e., the icosahedral and cuboctahedral structures. 
a)
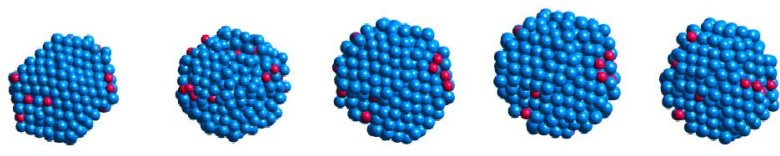

b)
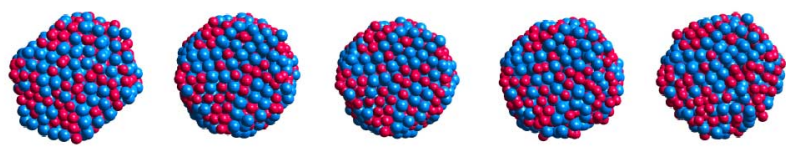

c)
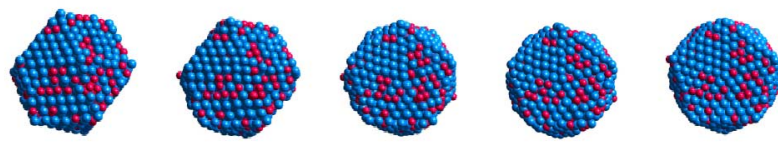

d)
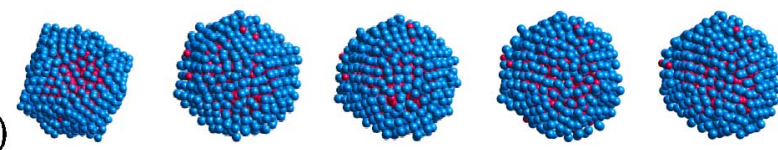

FIG. 5 (color online). $\quad N V T$ molecular dynamics simulations at $T=450 \mathrm{~K}$ of binary (blue/red) $\mathrm{AuCu}$ alloy clusters at different concentrations and particle sizes. Note how the component alloy concentration influences the structure of the particle. (a) $\mathrm{AuCu}$ particle alloy with 561 atoms at $10 \% \mathrm{Cu}$ concentration. (b) The same as (a) with increased $\mathrm{Cu}$ concentration at 50\%. (c) Particle with 923 atoms and $25 \% \mathrm{Cu}$ concentration. (d) The same as (c) with increased Cu concentration at $50 \%$.

particle with a (pseudo)fivefold axis of symmetry. This result is consistent with our experimental observation that reveals less than $1 \%$ of cubo-octahedral particles in the samples, which is in agreement with the observations of Koga and Sugawara [25] for pure Au clusters, and Saha et al. [26] for AuFe alloy with $25 \% \mathrm{Fe}$ concentration.

We have found that binary nanoparticles can present a number of nonperfect decahedral structures and the most novel is the one reported in Fig. 2 that corresponds to a decahedral structure with an atom (or a number of atoms [25]) missing on the center and the fivefold edges truncated. The structure results from the formation of a fault on the particle packing, where in the last layer a stacking fault is produced. The hcp surface atomic arrangement $(\ldots A B C B)$ of the tetrahedrons forming the decahedral particle (which regularly follows an fcc tetrahedral packing in monatomic clusters) evidenced some $\{100\}$ facets along the fivefold edges. This packing minimizes the surface free energy contribution in the small size regime ( $\leq 3800$ atoms) according to EAM calculations.

In summary, nonperfect decahedral shape and structure stabilization by surface reconstruction are reported in bimetallic AuPd and $\mathrm{AuCu}$ nanoparticles (1.5-3 nm). A number of new structures are found when two different metallic atoms are present. These novel structures present surface vacancy sites and the formation of a fault on the particle packing. The hcp surface atomic arrangement (...ABCB) evidenced some $\{100\}$ facets along the fivefold edges. This packing minimizes the surface free en- ergy contribution and favors the formation of these structures, according to our energy calculations and some others results reported previously. The formation of facets $\{100\}$ additional to the facets $\{111\}$ present in decahedral particles would have important implications in heterogeneous catalysis, because these new faces are chemically reactive and bonding sites for molecules, being specially important on catalytic systems such as $\mathrm{AuPd}, \mathrm{AuCu}$, and PdPt.

J. M. M. C. acknowledges financial support from CONACyT, México, through Grant No. G-25851-E. The authors are grateful to L. Rendón for technical assistance, with "The Texas Advanced Computing Center (TACC-UT)" for computing time on the IBM Power4 system, and Dr. Martín E. García and Dr. Aldo $\mathrm{H}$. Romero for careful reading of the text.

*Corresponding author.

Electronic address: yacaman@che.utexas.edu

[1] T. P. Martin, Phys. Rep. 273, 199 (1996).

[2] D. R. Nelson, Phys. Rev. Lett. 50, 982 (1983).

[3] J. P. K. Doye and D. J. Wales, Phys. Rev. Lett. 86, 5719 (2001).

[4] A. Mackay, Acta Crystallogr. 15, D916 (1962).

[5] L. D. Marks, Philos. Mag. A 49, 81 (1984).

[6] R. H. Leary and J.P. K. Doye, Phys. Rev. E 60, 6320 (1999).

[7] J. A. Ascencio et al., Surf. Sci. 447, 73 (2000).

[8] S. A. Nepigko et al., J. Cryst. Growth 213, 129 (2000).

[9] P. Gao et al., Scr. Metall. 22, 683 (1988).

[10] L. D. Marks and D. Smith, J. Microsc. 130, 249 (1982).

[11] V. G. Gryaznov et al., Phys. Status Solidi B 167, 441 (1991).

[12] Y. Chushak and L. S. Bartell, Eur. Phys. J. D 43, 46 (2001).

[13] C. L. Cleveland et al., Phys. Rev. Lett. 79, 1873 (1997).

[14] I. Srnová-Šloubová et al., Langmuir 16, 9928 (2000).

[15] S. Iijima and T. Ichihashi, Phys. Rev. Lett. 56, 616 (1986).

[16] P. M. Ajayan and L. D. Marks, Phys. Rev. Lett. 60, 585 (1988); 63, 279 (1989).

[17] C. Mottet, G. Tréglia, and B. Legrand, Surf. Sci. 383, L797 (1997).

[18] J. L. Rodríguez-López et al., Appl. Surf. Sci. 219, 56 (2003).

[19] J. F. Sánchez-Ramírez et al., J. New Mater. Electrochem. Appl. (to be published).

[20] A. P. Sutton and J. Chen, Philos. Mag. Lett. 61, 139 (1990).

[21] H. Raffi-Tabar and A. P. Sutton, Philos. Mag. Lett. 63, 217 (1991).

[22] S.C. Hendy and B. D. Hall, Phys. Rev. B 64, 085425 (2001); S. C. Hendy and J. P. K. Doye, ibid. 66, 235402 (2002).

[23] T. Radetic et al., Phys. Rev. Lett. 89, 085502 (2002).

[24] S. M. Foiles et al., Phys. Rev. B 33, 7983 (1986).

[25] K. Koga and K. Sugawara, Surf. Sci. 529, 23 (2003).

[26] D. K. Saha et al., Eur. Phys. J. D 9, 539 (1999). 